\title{
Fenomeno de Raynaud secundario y vasculitis cutànea asociado al uso de interferon: reporte de un caso.
}

\author{
Seeondary Raynaud's phenomenonant \\ eutaneous vaseullitis assoeiated with interferen: a \\ ease report.
}

\author{
Mario Andrés Hernández, José Ricardo Ruiz, Diego Vicente Lizarazo • \\ BogotÁ, D.C. (Colombia)
}

\begin{abstract}
Resumen
Se presenta el caso de un hombre adulto con fenómeno de Raynaud secundario y refractario al manejo médico asociado a vasculitis cutánea, que previamente venía recibiendo interferón beta para tratamiento de esclerosis múltiple. La agresividad del proceso requiere la interrupción de la medicación, la utilización de vasodilatadores, inhibidores de endotelina 1, simpatectomía bilateral por video toracoscópia, aplicación de toxina botulínica peri-arterial interdigital en manos y terapia inmunosupresora con corticoide a altas dosis y ciclofosfamida obteniendo detención del proceso isquémico, pero con pérdida anatómica asociada. (Acta Med Colomb 2014; 39: 00-00).
\end{abstract}

Palabras clave: fenómeno de Raynaud, interferón, autoinmunidad, vasculitis cutánea,

\begin{abstract}
A ease of an adtut mate with seeondary Raynatd's phenomenon refraetory to medieal treatment with associated eutaneous vaseulitis, that previously had been reeeiving interferon beta for multiple selerosis treatment. The aggressiveness of the proeess requires the interruption of the medieation, the use of vasodilators, inhibitors of endothelin-1, bilateral sympatheetomy by video thoracoseopy, bottlintm toxin in the hands Interdigitalperiatterial and immmosuppressive therapy with eortieosteroids and eyelophosphamide at high dosesobtaining eessation of ischemia, but with anatomieal loss associated. (Aeta Med Colomb 2014; 39:00-00).

Keywords: Raynatd phenomenon, interferon, antoimmunity, eutaneous vaseulitis.
\end{abstract}

\author{
Dr. Mario Andrés Hernández Sómerson: Re- \\ sidente Tercer Año de Medicina Interna; Dr. \\ Diego Vicente Lizarazo Hurtado: Residentes \\ Segundo Año de Medicina Interna; Dr. José \\ Ricardo Ruiz Cabrera: Residente Segundo \\ Año de Dermatología. Universidad el Bos- \\ que, Hospital Universitario Fundación Santa \\ Fe de Bogotá. Bogotá, D.C. (Colombia). \\ Correspondencia. Dr. Mario Andrés Hernán- \\ dez Sómerson. Bogotá, D.C. (Colombia). \\ E-mail: somerson18@hotmail.com, \\ somerson18@gmail.com \\ Recibido: 9/X/2012 Aceptado: 16/X/2013
}

\section{Introducción}

El fenómeno de Raynaud es causado por vasoespasmo episódico e isquemia de las extremidades y otras zonas distales en respuesta a estímulos emocionales y al frío. Puede ser primario o secundario a una condición subyacente y hasta en un $20 \%$ de los casos, puede ser la primera manifestación de enfermedades del tejido conectivo. Algunos medicamentos se han relacionado a su aparición, principalmente: derivados del ergot, beta bloqueadores y metisergida, siendo la relación con drogas biológicas rara vez reportada.

El interferón tipo I, se utiliza en la práctica clínica hace más de dos décadas, múltiples patologías incluyendo esclerosis múltiple, hepatitis $\mathrm{B}$ y $\mathrm{C}$ y procesos neoplásicos como linfoma folicular, sarcomas y melanomas son susceptibles de tratamiento con esta molécula. Su uso ampliado, ha llevado consigo la aparición de efectos adversos muy variables, comprometiendo prácticamente la totalidad de los sistemas corporales e incluso la inducción de procesos autoinmunes y vasoespásticos, cuyo impacto en la calidad de vida y los costos aún no ha sido medido.

$\mathrm{Al}$ revisar la literatura, existen pocas publicaciones de fenómenos de Raynaud y su relación con interferón. Se reporta un caso al respecto y se realiza una discusión con énfasis en los mecanismos fisiopatológicos y los resultados de las series publicadas.

\section{Presentación del caso}

Se presenta el caso de un paciente masculino de 72 años y de raza mestiza, que consulta al servicio de urgencias en mayo de 2012 con cambios en la coloración de los dedos de 
ambas manos dados por palidez seguida de cianosis e hiperemia, asociado a dolor y parestesias de un mes de evolución.

Como antecedente de importancia el paciente presentaba diagnóstico de esclerosis múltiple desde 10 años atrás, manejado desde entonces con interferón beta 1 alfa a dosis de 44 microgramos cuatro veces por semana, que requirió - tres meses antes - ajuste a dos dosis semanales por elevación de transaminasas.

Al examen físico de ingreso con evidencia clara de fenómeno de Raynaud en ambas manos, ingurgitación yugular grado II, velamiento de ruidos cardiacos y disminución del murmullo vesicular en ambas bases con edema grado II en miembros inferiores. Mediante tomografía de tórax y ecocardiograma se evidencia: derrame pleural bilateral y derrame pericárdico de $650 \mathrm{cc}$ con signos de taponamiento cardíaco, ambos compatibles con exudado linfocítico, Se realiza perfil de autoinmunidad, toracentesis y pericardiocentesis, cuyos resultados se destacan en las Tabla 1 y 2 , ecografía doppler arterial de miembros superiores no evidencia estenosis significativas. Por su asocio con inducción de autoinmunidad se suspendió interferón y se consideró como causa autoinmune la presencia de serositis, por lo cual se inició manejo con bolos de metilprednisolona de $250 \mathrm{mg}$ intravenosos por tresdías. Presenta mejoría paulatina de edemas periféricos y derrames, con persistencia de fenómeno de Raynaud a

Tabla 1. $x x x x x x$

\begin{tabular}{|l|c|c|}
\hline Laboratorio & Valor & Resultado \\
\hline Factor reumatoide & $67.5 \mathrm{UI} / \mathrm{mL}$ & Positivo \\
Proteína C reactiva & $6 \mathrm{mg} / \mathrm{dL}$ & Elevada \\
VSG & $44 \mathrm{~mm} / \mathrm{hora}$ & Elevada \\
Anticuerpos anti nucleares & $1: 1280$ patrón moteado & Positivo \\
Complemento sérico C3 & $88.3 \mathrm{mg} / \mathrm{dL}$ & Normal \\
Complemento sérico C4 & $24.2 \mathrm{mg} / \mathrm{dL}$ & Normal \\
Anticuerpos anti RO (EIA) & 97.3 & Positivo fuerte \\
Anticuerpos anti LA (EIA) & 70.7 & Positivo fuerte \\
Anticuerpos anti Sm y anti DNA dc & Negativo & Negativo \\
Criogllbulinas & Negativo & Negativo \\
Anticuerpos anti cromatina & Negativo & Negativo \\
Anticuerpos anti-citoplasma de neutrófilos & Negativo & Negativo \\
Anticuerpos anti-cardiolipinasIg G e Ig M & Negativos & Negativos \\
Anticuerpos anti B2 GP-1 & Negativo & Negativo \\
Anticoagulante lúpico & Negativo & Negativo \\
\hline
\end{tabular}

Tabla 2. $x x x x x x x x$

\begin{tabular}{|l|c|c|}
\hline Laboratorio & Valor & Resultado \\
\hline Factor reumatoide & $21.2 \mathrm{UI} / \mathrm{mL}$ & Positivo \\
Complemento C3 & $46.3 \mathrm{mg} / \mathrm{dL}$ & Disminuido \\
Complemento C4 & $9-9 \mathrm{mg} / \mathrm{dL}$ & Disminuido \\
Anticuerpos anti-nucleares & $1: 1280$ patrón granular & Positivo \\
\hline
\end{tabular}

pesar de manejo vasodilatador con nifedipino a dosis de 60 miligramos diarios y losartán a dosis de 200 miligramos diarios, episodios sostenidos y de duración prolongada de cianosis distal y posterior aparición de úlceras digitales y necrosis en las primeros cinco días luego de su ingreso, a nivel de segundo y quinto dedo de la mano derecha y segundo y cuarto dedo mano izquierda (Figura 1). Se adiciona manejo con sildenafil a dosis progresiva hasta $100 \mathrm{mg}$ diarios y se realiza biopsia de piel a nivel del pulpejo de segundo dedo de la mano derecha reportando hallazgos compatibles con fenómeno de Raynaud y vasculitis cutánea (Figura 2), la video capilaroscopia reporta alteración severa de la arquitectura capilar sin patrón definido. Con clara evidencia de proceso autoinmune dados por paraclínicos y biopsia de piel se continúa prednisolona a $1 \mathrm{mg} / \mathrm{Kg} /$ día y ciclofosfamida $500 \mathrm{mg}$ intravenosos quincenales por cuatro dosis. Ante persistencia de isquemia digital se decide realización de simpatectomía bilateral por video- toracoscópia, la cual se realiza al séptimo día de hospitalización, obteniendo leve mejoría de isquemia digital a las 48 horas de la intervención, pero con posterior recrudecimiento del proceso, por

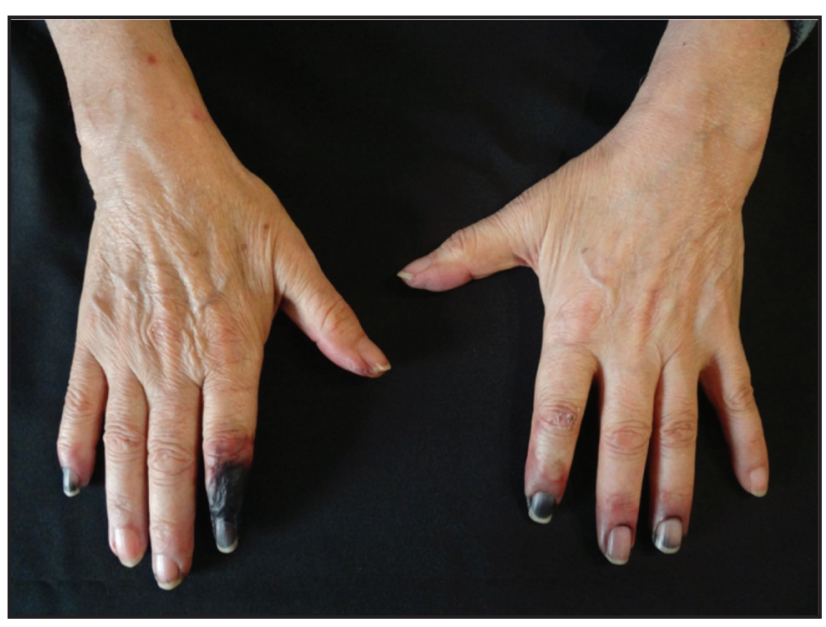

Figura 1. Fenómeno de Raynaud. Isquemia y necrosis distal en dedos de la mano.

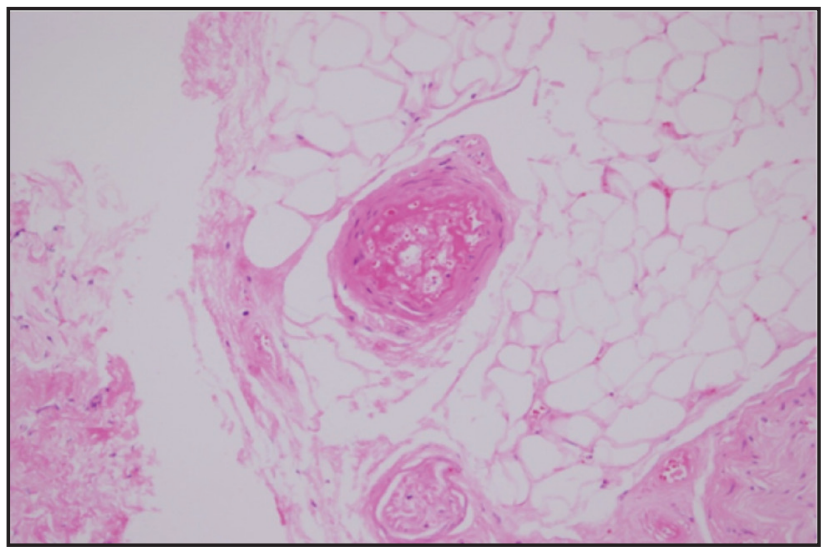

Figura 2. Microfotografía a 40X, tinción con hematoxilina y eosina. Necrosis fibrinoide de áreas vasculares de pequeño y mediano calibre. 
lo que es llevado a inyección interdigital periarterial de toxina botulínica en ambas manos, hasta dosis de 100 UI. Se adiciona manejo con bosentàn a dosis de $62.5 \mathrm{mg}$ cada 12 horas. Luego de 15 días del manejo inmunosupresor se evidencia clara delimitación de la isquemia dejando como secuela necrosis de falange distal y media del segundo dedo y del pulpejo de quinto dedo de la mano derecha con necesidad de amputación.

\section{Discusión}

Los interferones fueron descritos inicialmente por Isaacs y Lidenmann en 1957 y su nombre fue acuñado gracias a la capacidad de interferir con la replicación viral en cultivos celulares (1). Existen tres tipos de interferones (I-III), entre los cuales la familia tipo I ha sido ampliamente estudiada y sus efectos los más conocidos, se subdivide en cinco grupos: alfa, beta, mi, épsilon y kappa, de ellos los dos primeros son los más utilizados en la práctica clínica (2). Los interferones constituyen el principal mecanismo de defensa contra la infección viral, pero a su vez tiene funciones inmunoduladoras produciendo activación general del sistema inmune, inducción de la maduración y activación de células presentadoras de antígenos, aumento en los niveles de muchas citoquinas y sus receptores, así como de moléculas co-estimuladoras, también se han encontrado en la vía de activación de linfocitos ayudadores y citotóxicos (3).

Desde 1980, se iniciaron los estudios clínicos que validad su uso en múltiples patologías, principalmente de tipo infeccioso, pero también en neoplasias hematológicas y como en el caso reportado, en esclerosis múltiple $(2,4)$. El interferón alfa se encuentra disponible como interferón alfa- $2 \mathrm{a}$, alfa 2 a pegilado, alfa $2 \mathrm{~b}$ y alfa $2 \mathrm{~b}$ pegilado, mientras el interferón beta es comercialmente disponible como beta 1a y beta $1 \mathrm{~b}$ (2). El interferón alfa, se une 1000 veces con mayor afinidad a su receptor que el interferón beta, por lo cual los efectos adversos, especialmente autoinmunes se observan en mayor asociación al primero (5). Dentro de los efectos adversos de la medicación encontramos principalmente fiebre, artralgias, mialgias, escalofríos como los más comunes y menos frecuencia tenemos: convulsiones, alteración del estado de conciencia, síntomas gastrointestinales, dolor músculo esquelético y arritmias cardíacas, angina e infarto agudo de miocardio $(2,5)$. Su relación a la inducción de enfermedades autoinmunes durante la terapia se encuentra demostrada, siendo la enfermedad tiroidea la más común, seguida de lupus eritematoso sistémico, sarcoidosis y vitíligo. La presencia de fenómeno de Raynaud y otros fenómenos vasculares constituyen un efecto adverso de creciente reporte como se describirá a continuación (6).

Schapira y colaboradores, reportan la serie más grande descrita en la literatura de asociación entre uso de interferón y fenómeno de Raynaud, 24 casos en total, encontrados desde 1967 hasta el año 2001. El uso de interferón beta se documentó en 10 pacientes, con síntomas que variaban desde leve vasoespasmo hasta isquemia y necrosis digital con necesidad de amputación en seis casos. La aparición de fenómeno de Raynaud se produjo desde pocas semanas luego del uso del medicamento hasta incluso 10 años después y la recuperación parcial o completa solo pudo demostrarse en siete casos (7).

Al-Zahranl y colaboradores, reportan 13 casos de pacientes con neoplasias hematológicas en manejo con interferón alfa que presentaron fenómenos vasculares entre la tercera semana y los tres primeros años desde su inicio, siendo los más frecuentes la isquemia digital, necrosis de los dedos y el fenómeno de Raynaud, encontrando también condiciones menos frecuentes como microangiopatía trombótica y vasculitis pulmonares probadas con biopsia. En esta serie todos los pacientes requirieron terapia inmunosupresora (corticoides y ciclofosfamida) para el control de los síntomas (6).

Los mecanismos por los cuales la terapia con interferones tipo I induce fenómenos vasculares no están bien dilucidados. Los interferones tipo I y tipo II, están claramente implicados en la autoinmunidad. Por la vía de estímulos a receptores Toll-Like pueden activarse células presentadoras de autoanticuerpos, llevando a activación células $\mathrm{T}$ autoreactivas, este estímulo igualmente activa a células $\mathrm{B}$, promoviendo la expresión de autoanticuerpos reactivos contra complejos DNA-RNA. Las señales de interferón alfa y beta promueven y amplifican la activación de linfocitos $\mathrm{T}$ y B, además de aumentar la expresión de otras citoquinas y moléculas de complejo mayor de histocompatilibidad clase I y II (6). Otros mecanismos propuestos incluyen: efecto vasoespástico directo del medicamento, activación de linfocitos T reactivos contra células endoteliales, estimulación de la angiogénesis e incluso actividad pro-coagulante resultando en oclusión arterial por trombos (5).

En el caso particular del paciente presentado, la aparición de los síntomas se dio luego de tres años de aplicación de interferón beta- 1alfa, con sintomatología muy agresiva predominantemente isquémica y necrosis digital asociada a proceso vasculítico. Llama la atención, la elevación de auto-anticuerpos (anti RO, anti LA y ANAS) y compromiso seroso asociado (este hallazgo no reportado previamente en la literatura), con alta probabilidad de tratarse de lupus inducido por medicamento. Aún más, bastante llamativo la severidad de la manifestación llevando a la pérdida anatómica en un período corto de tiempo, a pesar del manejo vasodilatador e inmunosupresor, lo cual constituye la minoría de los casos reportados.

El manejo del fenómeno de Raynaud refractario constituye un reto médico por la latente posibilidad amputación que conlleva su presencia $(8,9)$. El manejo específico de los casos asociados a uso de interferón, no difieren del manejo habitual. En la gran mayoría de las series, el retiro de la droga y terapia vasodilatadora resulta eficaz en los casos leves, siendo los casos refractarios requirentes de medidas intervencionistas e incluso terapia inmunosupresora, máxime cuando se asocia a vasculitis de cualquier tipo.

La aplicación de toxina botulínica periarterial interdigi- 
tal merece espacial atención. Es una técnica cada vez más utilizada como tratamiento de casos severos y refractarios. Se ha propuesto como mecanismo de acción el bloqueo del vasoespasmo por inhibición de la vasoconstricción inducida por frío y por la prevención de reclutamiento de receptores alfa 2 en el musculo liso vascular en condiciones de frío. Iorio y colaboradores revisan cinco estudios publicados hasta el año 2010, que incluían pacientes con dolor isquémico, úlceras digitales y resistencia al manejo médico, la dosis de toxina botulínica utilizada estuvieron entre 10 y 100 UI con mejoría de dolor entre un $75 \%$ y $85 \%$ y mejoría de úlceras hasta en el $100 \%$. Como complicación principal: debilidad de musculatura intrínseca hasta en el $27 \%$ de los casos, se requieren ensayos clínicos controlados para probar su eficacia (10).

\section{Conclusiones}

Aunque la presencia de enfermedades autoinmunes asociadas al uso clínico de interferón se considera un evento adverso conocido, creciente evidencia se acumula respecto a la inducción de procesos vasculares, incluyendo fenómeno de Raynaud y vasculitis como manifestación rara de efecto adverso a esta medicación, por lo cual, debe considerarse siempre que se pretenda utilizar para cualquier patología.
El manejo de estos casos, varía dependiendo de la severidad del proceso, siendo el retiro del medicamento obligatorio y la terapia vasodilatadora, la pieza angular, dejando la terapia intervencionista e inmunosupresión para los casos refractarios o asociados a patología autoinmune demostrada.

\section{Referencias}

1. ChoubeyD, Mougdil KD. Inteferons in autoimmune and inflammatory diseases: regulation and roles. J Interferon Citokine Res 2011; 31(12): 857-865.

2. Burdick LM, Somani M, Somani AK. Type I IFNs and their role in the development of autoimmune disease. Expert Opin Drug Saf 2009; 8(4): 459-472.

3. Biggiioggero M, Gabbriellini L, Meroni PL. Type I interferon therapy and its role in autommunity. Autoimmunity 2010; 43(3): 248-254

4. Baccala R, Kono DH, Thephilopoulos AN. Interferons as pathogenic effectors in autoimmunity. Immunological Reviews 2005; 204: 9-26.

5. Sozzani S, Bosisio D, Scarsi M. Type I interferon and systemic autoimmunity. Autoimmunity 2010; 46 (3): 196-203.

6. Al-zahranl H, Gupta V, Minden HA. Vascular events associated with alpha interferón therapy. Leukemia \& lymphoma 2003; 44 (3): 471-475.

7. Schapria D, Nahir AM, Hadad N. Interferon-induced Raynaud`s syndrome. Sem Arthritis Rheum 2002; 32: 157-162.

8. Baumhäkel M, Bönm M. Recent achievements in the management of Raynaud`s phenomenon. Vasc Healt Risk Manag 2010; 6: 207-214.

9. Leiven Tl. Advances in the treatment of Raynaud`s phenomenon. Vasc Healt Risk Manag 2010; 6: 167-177.

10. Iorio ML, MasdenDI, Higgins JP. Botulinumtoxina A treatment of Raynaud`s phenomenon: a review. Sem Arthritis Rheum 2012; 41: 599-603. 